\title{
The Intracellular Journey of Shiga Toxins
}

\author{
Maria L. Torgersen ${ }^{1}$, Nikolai Engedal ${ }^{1}$, Jonas Bergan ${ }^{1}$ and Kirsten Sandvig*, ${ }^{* 1,2}$
}

${ }^{1}$ Centre for Cancer Biomedicine, Institute for Cancer Research, Faculty Division: The Norwegian Radium Hospital,
University of Oslo, Montebello, 0310 Oslo, Norway; ${ }^{2}$ Department of Molecular Biosciences, University of Oslo, 0316
Oslo, Norway

Abstract: The Shiga toxin family consists of Shiga toxin (Stx) that is produced as a virulence factor by Shigella dysenteriae, and the Shiga-like toxins produced by certain strains of enterohemorrhagic E. coli as well as by some other types of bacteria. Infection with bacteria producing these toxins is a threat to human health even in industrialized countries, as the initial diarrhea caused by the infection might be followed by a complication named hemolytic uremic syndrome. The Shiga toxins consist of a binding moiety that in most cases binds to the glycosphingolipid Gb3 on the surface of susceptible cells, and an A-moiety responsible for the toxic effect in the cytosol. In order to reach its cytosolic target, the toxin must be internalized and then transported via the retrograde pathway to the Golgi complex and further to the endoplasmic reticulum. From the endoplasmic reticulum the enzymatically active part of the A-moiety is translocated to the cytosol, and cellular protein synthesis is inhibited. Although the Shiga toxins are involved in disease, they may also be exploited for medical diagnosis and treatment. Interestingly, the toxin receptor, $\mathrm{Gb} 3$, has a limited expression in normal tissues, but is overexpressed in several types of cancer. Thus, the use of Shiga toxin, or the binding part of the toxin, has great potential in cancer diagnostics and treatment. Furthermore, studies of the various uptake mechanisms and intracellular transport pathways exploited by the toxins, provide important insight in basic cell biology processes.

Keywords: Shiga toxin, retrograde transport, endocytosis, Gb3, glycosphingolipids, trans-Golgi network.

\section{INTRODUCTION}

The Shiga toxins are $\mathrm{AB}_{5}$-toxins consisting of a pentameric binding moiety (StxB) and an enzymatically active A-moiety (StxA) (Fig. 1). The 5 small B-chains (Mw $7.7 \mathrm{kD}$ ) interact non-covalently, and each B-chain contains three binding sites for the glycosphingolipid Gb3 [1]. Thus, the pentamer has in theory the ability to cluster up to $15 \mathrm{~Gb} 3$ molecules, which is important for toxin binding (see below). Only one type of Shiga-like toxin has been found to bind Gb4. The A-moiety, with a molecular mass of $32.2 \mathrm{kD}$, is non-covalently attached to the B-moiety [2] (Fig. 1), and contain a loop formed by a disulfide bond between cysteines 242 and 261.

An important step for intoxication with Shiga toxin is the cleavage of the A-chain by the protease furin, generating the enzymatically active $A_{1}$ fragment that is able to translocate into the cytosol [3]. Furin cycles between the TGN and the cell surface, and is involved in cleavage and activation of not only Shiga toxin, but also diphtheria toxin and Pseudomonas exotoxin A [4]. The pH-optimum for toxin cleavage varies with the substrate, and for Shiga toxin, furin-induced cleavage has a low pH-optimum [5], indicating that efficient cleavage can occur shortly after endocytosis. After cleavage, the $A_{1}$ fragment remains attached to the $A_{2}$ part via the internal disulfide bond [5], and the cleaved toxin is transported further via the Golgi complex to the endoplasmic reticulum (ER). From the $E R$, the $A_{1}$-fragment is

*Address correspondence to this author at the Centre for Cancer Biomedicine, Institute for Cancer Research, Faculty division, The Norwegian Radium Hospital, University of Oslo, Montebello, 0310 Oslo, Norway Tel: +47 22781828; Fax: + 47 22781265;

E-mail: ksandvig@radium.uio.no translocated to the cytosol and functions as a highly specific $\mathrm{N}$-glycosidase that removes adenine from one particular adenosine residue in the 28S RNA of the 60S ribosomal subunit [6]. The depurinated subunit is unable to interact properly with elongation factors, and the protein synthesis is halted. This mechanism of action is identical for the Shiga toxins and several plant toxins, e.g. ricin and abrin [7], indicating that important features of these toxins are conserved.

The Shiga-like toxins are divided into two immunologically distinct groups. Shiga-like toxin 1 (Stx1) is virtually identical to Stx produced by Shigella dysenteriae and differs in only one amino acid in the A-chain. Shiga-like toxin 2 (Stx2) shares the overall toxin structure, but has lower amino acid similarity to Stx ( $55 \%)$. There are several subtypes of Stx2, and some bacteria produce several types of Shiga-like toxins [8]. Although Stx 1 is 5-10 fold more cytotoxic in vitro than Stx2 [9], Stx2 is most frequently associated with human disease, such as the hemolytic uremic syndrome (HUS). This condition is defined by acute renal failure, hemolytic anemia and thrombocytopenia, and most frequently affects children and elderly (reviewed in [10]). Potential sources of the toxin-producing bacteria are undercooked ground beef, unpasteurized milk and juice, raw vegetables, and contact with infected live animals.

In some cell types the Shiga toxins, as a long term effect, induce expression and secretion of pro-inflammatory cytokines, including TNF- $\alpha$, IL-1, IL-6 and IL-8 $[6,11,12]$. Interestingly, the secreted cytokines can induce synthesis of $\mathrm{Gb} 3$ in other cell types, thereby sensitizing these cells to the toxin [13]. The Shiga toxins are also known to induce apoptosis either via the ribotoxic stress induced after transport of the A-chain into the cytosol, or, as shown in 


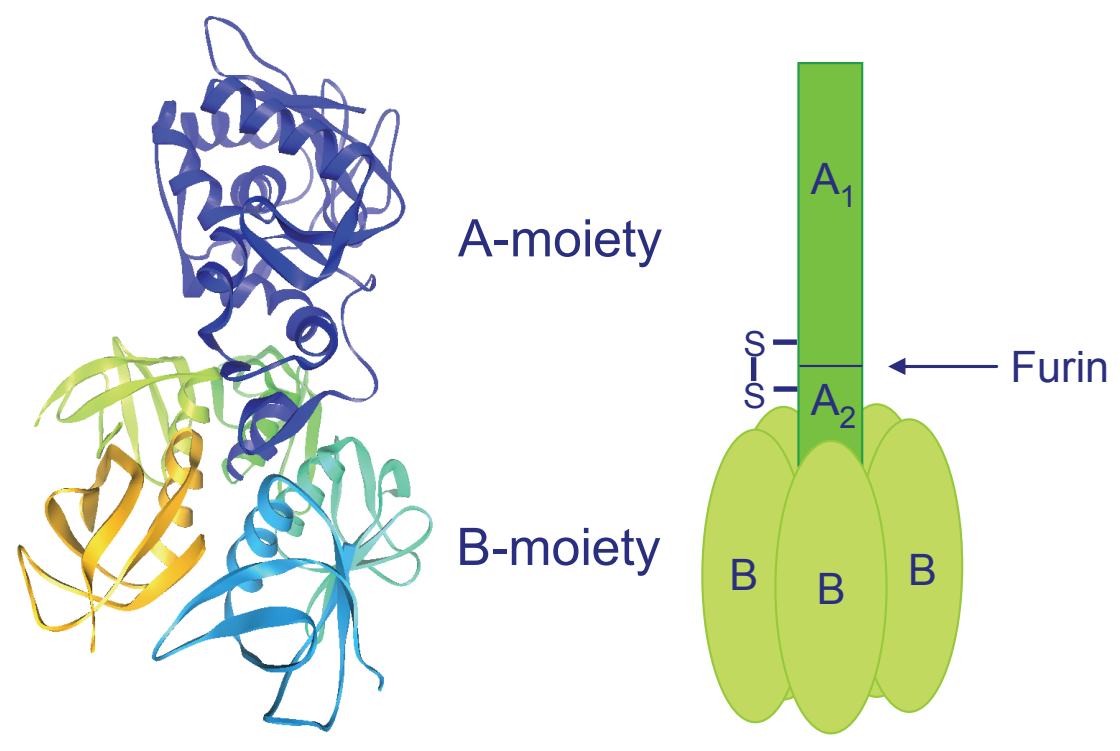

Fig. (1). The structure of Stx. Stx (PDB protein data bank: 1DMO) consists of an A-chain of $\sim 32 \mathrm{kDa}$, non-covalently attached to the ringshaped, pentameric B-moiety. The five B-chains of $7.7 \mathrm{kDa}$ each, spontaneously assemble into the pentameric form. The A-chain is activated by proteolytic processing during the intracellular trafficking in the target cell. The protease furin has been shown to nick the A-chain into an enzymatically active $\mathrm{A}_{1}$-fragment $(\sim 27 \mathrm{kDa})$ and a carboxyl terminal $\mathrm{A}_{2}$-fragment $(\sim 4 \mathrm{kDa})$, which remain linked by a disulfide bond.

Burkitt lymphoma cells, by rapid signaling induced at the cell surface [11, 14-16].

\section{BINDING OF STX TO CELLULAR MEMBRANES}

All the members of the Shiga toxin family, except Stx2e, bind to the oligosaccharide part of the glycosphingolipid Gb3, with the structure $\mathrm{Gal}(\alpha 1-4) \mathrm{Gal}(\beta 1-4)$ GlcCer. Stx2e binds to $\mathrm{Gb} 4$ and is produced by bacteria that mainly infect pigs. Although Stx 2 is most frequently associated with human disease, in most cases Stx 1 has been shown to bind more strongly than Stx2 to Gb3 [17-20]. When the crystal structure of Stx 2 and Stx is compared, some differences are found that might influence the ability of the toxins to interact with human cells and cause disease [21]. In contrast to Stx, the active site of the Stx2 A-moiety is accessible in the holotoxin, the orientation of the A-chain with respect to the B-pentamer differs in the two toxins, and one of the three Gb3 binding sites in the Stx2 B-chain has a different conformation than in Stx B-chain.

Although the toxin binds to the oligosaccharide part of $\mathrm{Gb3}$, it is known that also the structure of the lipid moiety of the receptor is important for toxin binding, most likely by affecting the presentation of the oligosaccharide part. Both hydroxylation, the degree of unsaturation of the fatty acid, and the fatty acid chain length are important factors for optimal toxin binding $[19,22,23]$. The importance of the fatty-acid chain length of Gb3 for Shiga-like toxin binding has recently been found to differ for Stx1 and Stx2 [24]. In a model membrane system Stx1 bound selectively to C16, $\mathrm{C} 22$, and $\mathrm{C} 24 \mathrm{~Gb} 3$-species, but not to $\mathrm{C} 18$ or $\mathrm{C} 20$ species. In contrast, Stx2 bound to all isoforms of $\mathrm{Gb} 3$, irrespective of chain length.

The structure of $\mathrm{Gb} 3$ is not the only parameter that affects Shiga toxin binding; also the local membrane environment surrounding $\mathrm{Gb} 3$, such as the phospholipid- and cholesterol-content of the membrane is important $[9,20,23$,
25]. Also the density of Gb3 in the membrane seems to affect Shiga toxin binding, as it was recently found that mutant Vero cells with a lower density of $\mathrm{Gb} 3$ were unable to bind Stx1 [26]. Notably, at least in HeLa cells, toxin binding seems to induce recruitment of the toxin/receptor complex to lipid microdomains, or rafts, in the plasma membrane [27], and this localization has been reported to be important for correct retrograde trafficking and toxicity (see below). Recently, it was demonstrated that Stx1 and Stx2 (also called Verotoxin 1 and 2) localized to both shared and distinct microdomains on the cell surface, and Stx1 was more resistant to detergent extraction than Stx 2 both at the cell surface and during intracellular transport [9]. From this it seems that the toxin homologs bind different Gb3 lipid assemblies. Moreover, during their retrograde transport, the two toxins showed both overlapping and distinct localization, and also the kinetics of their retrograde transport differed [9]. These factors might contribute to the differential effects on target cells that these toxins display.

Interestingly, it has been shown that Stx binding rapidly activates several kinases, such as the Src kinases Yes [28, 29] and Lyn [30], the tyrosine kinase Syk [30, 31], the serine/threonine kinase PKC $\delta$ [32], and the MAP kinase p38 $\alpha$ [33]. The activity of some of these kinases is important for Stx entry and intracellular transport, suggesting that Stx is able to induce its own transport (see below).

\section{ENDOCYTOSIS OF STX}

Stx is able to exploit several endocytic mechanisms to gain entry into the cell, and the toxin was the first lipidbinding ligand shown to utilize clathrin-dependent endocytosis [34]. The Gb3 molecules does not seem to be constitutively present in clathrin-coated pits, rather, the toxin/receptor complex is recruited into coated pits and internalized [34]. Interestingly, Stx seems to be able to stimulate its own clathrin-mediated uptake. The B-moiety is sufficient to activate Syk and induce phosphorylation of 
clathrin and uptake by this pathway [31], however, the efficiency of clathrin-mediated toxin uptake is dependent on the concentration of cell surface-bound toxin molecules and the presence of the toxin A-chain [35]. The stimulated uptake seen at high concentrations of intact toxin, might be explained by increased aggregation of toxin-receptorcomplexes into large clusters that somehow enhance the recruitment to clathrin-coated pits. Also, the toxin-receptor complex might interact with other plasma membrane proteins recruited to clathrin-coated pits. In fact, Stx has been shown to associate with surface-proteins in Vero and CaCo-2 cells [36], although the identity of these proteins remains unknown.

Interestingly, both the holotoxin and the B-moiety without an A-chain, can induce rapid tyrosine phosphorylation of several proteins, including the tyrosine kinase Syk and clathrin heavy-chain [31, 37]. Upon stimulation with Stx/StxB a complex between Syk and clathrin heavy-chain was detected, and in Ramos cells both the phosphorylation of clathrin and the complex-formation seemed to require Src family kinase activity [37]. Moreover, depletion or inhibition of Syk reduced Stx uptake, suggesting a link between Stx-induced signaling and endocytosis. Subsequently, Stx binding has been shown to activate other key kinases that have roles in its transport, namely $\mathrm{PKC} \delta$ [32] and $\mathrm{p} 38 \alpha$ [33]. These kinases do not seem to regulate the uptake of the toxin, but rather to be involved in the endosome-to-Golgi transport step (see below). Moreover, Stx has been shown to stimulate microtubule assembly in ACHN cells [38] and in Vero cells [39], and both microtubules and dynein were found to be required for transport of Stx to the Golgi [39]. Notably, it was shown that the Stx-induced activation of microtubuli assembly was not mediated via Syk, suggesting that multiple signaling pathways are induced by Stx.

Clathrin-mediated endocytosis can be responsible for a large fraction of the endocytic uptake of Stx [40], but also other, clathrin-independent, mechanisms have been shown to contribute to toxin internalization in different cells [41-45]. These clathrin-independent uptake mechanisms show different requirements for dynamin and lipid rafts. In HeLa cells, we find that $\sim 50 \%$ of Stx uptake is independent of dynamin [40], however, it is important to bear in mind that inhibition of one pathway might lead to upregulation of another [46]. It is therefore difficult to determine the exact contribution from each pathway in toxin uptake. Recently, a novel clathrin-independent uptake mechanism was reported for StxB, where the toxin was shown to induce tubular membrane invaginations mediating its uptake [45]. It was postulated that clustering of up to $15 \mathrm{~Gb} 3$ molecules by StxB binding generates cluster domains that naturally induce negative curvature on the membrane, and that this leads to invagination and generation of tubules [45]. The formation of the tubules was shown to be energy-independent, but dynamin was required for proper fission. To which extent this process is important for intoxication is not yet known.

\section{ENDOSOME-TO-GOLGI TRANSPORT OF STX}

Retrograde transport from endosomes to the TGN and further to the Golgi is important for retrieval and correct localization of several endogenous proteins, e.g. Golgi- resident proteins such as TGN46, mannose 6-phosphate receptors (MPRs) and furin (for review see [47]). Some pathogen-produced proteins, such as the Shiga toxins, cholera toxin, ricin and Pseudomonas exotoxin A, are able to exploit this pathway in order to reach their intracellular targets. After uptake into early endosomes, a key sorting process that determines the fate of the toxin takes place. The toxin/receptor complex might be sorted into the degradative pathway to the lysosomes or into the retrograde pathway to the TGN. Although, the sorting mechanism is currently not completely understood, several components that seem to play a role in this process have been revealed (Fig. 2).

Two main retrograde transport routes to the TGN have been identified; a direct pathway from early endosomes or the recycling compartment, and an indirect pathway via late endosomes [48-51]. Stx has been shown to utilize the direct pathway to the TGN, and not the Rab9-dependent pathway via late endosomes that transports amongst others, furin and a fraction of Pseudomonas exotoxin A [48, 52-55]. However, based on the differential requirements for retrograde transport of Stx, ricin and TGN38, it seems that more than one parallel pathway between early endosomes and the TGN may exist [32, 33, 56-62].

Several Rab- or Rab-associated proteins have been implicated in the transport of StxB from early endosomes to the TGN. Rab11 [60], Rab6a' [59, 63], and Rab6IP2 [64] have all been reported to positively regulate Stx transport, while Rab9 does not seem to be required for transport of either ricin or Stx $[55,56]$. In a screen for potential Rab GTPase activating proteins (RabGAPs) involved in transport of Stx to the Golgi, the Golgi-localized Rab43 and its RabGAP RN-tre were found to be required [65]. Additional 5 RabGAPs were identified as regulators of Stx transport to the Golgi, however, their target Rabs remain unknown.

Interestingly, both clathrin and dynamin play an important role not only in Stx uptake, but also in Stx transport from early endosomes to the Golgi [40, 44, 66]. The clathrin adaptor epsinR was found to be required for transport of Stx to the Golgi, while AP-1 did not seem to be involved [44]. Moreover, coat-like components other than clathrin, such as the retromer complex, has been shown to mediate transport of Stx from early endosomes to the Golgi [67-69] (Fig. 2). The retromer consists of the components hVps26, hVps29, hVps35, SNX1, and possibly SNX2 (for review see $[70,71])$, and this complex has been shown to mediate retrieval of MPRs [51,72] and several other cargo proteins to the Golgi [70]. Importantly, the retromer components $\mathrm{hVps} 26$ and SNX1, and also SNX2, are required for endosome-to-Golgi transport of Stx [67-69]. Sorting nexins contain a Phox homology $(\mathrm{PX})$ domain that binds to certain phosphoinositides, such as $\mathrm{PI}(3) \mathrm{P}$, and a BAR (Bin/Amphiphysin/Rvs) domain that senses or induces membrane curvature. The presence of $\mathrm{PI}(3) \mathrm{P}$ and the activity of the PI(3) kinase hVps34 is important for efficient transport of both Stx and ricin to the TGN [68, 73]. The retromer-interacting proteins EHD1 (Eps15 homology domain-containing protein 1) and EHD3 are needed for proper localization of SNX1 and SNX2 to endosomal tubules [74], and both EHD3 and one of its interaction partners, the Rab4/5 effector rabenosyn-5, are required for endosome-toGolgi transport of Stx [74]. How can we explain the 


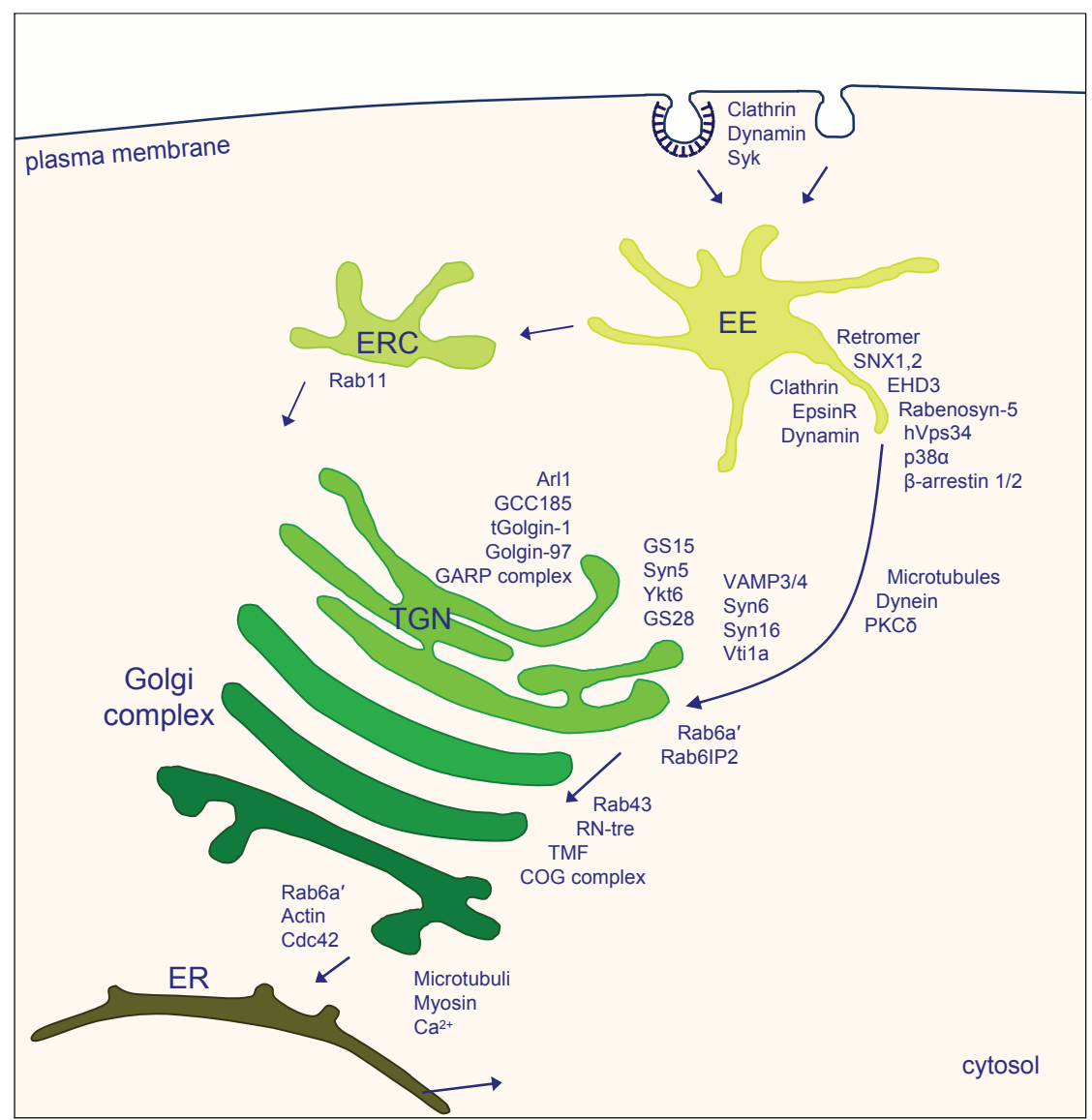

Fig. (2). Overview of components involved in the retrograde trafficking of Stx. Endocytosis of Stx is mediated by several uptake mechanisms, with different requirements for components such as clathrin, dynamin, and Syk. From early endosomes (EE) Stx is transported to the trans-Golgi network (TGN), either directly and/or via the endocytic recycling compartment (ERC). Several components have been implicated in the exit from EE and in the further retrograde transport. These are discussed in detail in the text.

requirement for both clathrin and the retromer complex in transport of Stx to the Golgi? One likely explanation is that the clathrin coat and the retromer complex are not independent of each other, but rather regulate the retrograde transport from endosomes sequentially.

Several tethering factors seem to be involved in retrograde transport of Stx. tGolgin-1 [75], Golgin-97 and its effector ARL1 [76, 77], the conserved oligomeric Golgi (COG)-complex [78], GCC185 [79], GARP (Golgiassociated retrograde protein) complex [80], and Rab6binding TATA element modulatory factor (TMF) [81] have all been implicated in Stx transport, presumably by targeting the Stx-containing vesicles to the TGN/Golgi. Moreover, two distinct SNARE complexes seem to be involved in the fusion of Stx containing vesicles with the TGN. One consists of the v-SNARE VAMP3/4 recognizing the t-SNARE complex syntaxin-6/syntaxin-16/Vtila $[59,77]$ and the other fusion complex consists of the v-SNARE GS15 and the tSNARE complex syntaxin-5/Ykt6/GS28 [82]. It has been speculated that ARL1 is functionally connected to the tSNARE complex, possibly by regulating the localization or function of the components Vtila and syntaxin-6 [77].

In several cell-types, exit from early endosomes into the retrograde pathway of both cholera toxin and Stx has been shown to depend on lipid raft integrity [83-89]. It is postulated that these toxins exploit an endogenous pathway for recycling of raft glycolipids and that the lipids mediate toxin transport all the way from the plasma membrane to the ER $[87,89]$. In agreement with this, disruption of lipid rafts or reduction of cellular cholesterol by drugs such as $\mathrm{m} \beta \mathrm{CD}$ or filipin, was found to strongly reduce transport of Stx to the Golgi $[55,87]$. However, not only lipid raft integrity, but also specific targeting of the Stx/Gb3 complex to these domains seems to be important for retrograde transport [87]. This was indicated by comparing Stx transport in toxin sensitive HeLa cells vs. toxin insensitive monocyte-derived cells [87]. The toxin was internalized in both cell types, but was only targeted to the Golgi in HeLa cells, corresponding to raft localization of Gb3 in these cells and not in the monocyte-derived cells. A correlation between raft localization and toxicity has also been shown by comparing cholera toxin and E. coli heat-labile enterotoxin IIb [89]. Both toxins were internalized, but only raft-localized toxinreceptor complexes were targeted to the Golgi and displayed cytotoxicity [89]. Moreover, bovine intestinal epithelial cells express Gb3, but are insensitive to Shiga-like toxins [90]. In these cells the internalized toxin is routed to lysosomes for degradation, like in monocyte-derived cells. Because of the insensitivity towards Shiga-like toxins, cattle function as reservoirs of Shiga-like toxin-producing bacteria [90]. 
Targeting of the Stx/Gb3 complex from endosomes to the Golgi appears to depend on the fatty acid chain length of Gb3 [62, 91-93]. Sensitization of cells towards Stx by treatment with byturic acid is known to cause a change in $\mathrm{Gb} 3$ fatty acid chain length, and Gb3 species containing C16 fatty acids may favor endosome-to-Golgi transport of Stx $[62,91,93,94]$. Recently, it was shown in HEp-2 cells that inhibition of glycolipid synthesis with PDMP or fumonisin $B_{1}$ results in loss of the glycolipid species at different rates [62]. Gb3 molecules with C16 fatty acids were degraded faster and to a larger extent than C24:1 Gb3 molecules. The
endosome-to-Golgi transport was inhibited and the toxin seemed to end up in endosomes or transport vesicles unable to fuse with the Golgi complex. Neither treatment with PDMP [62] nor fumonisin $B_{1}$ (Fig. 3) resulted in toxin transport to LAMP1-positive late endosomes/lysosomes. PDMP treatment changed the cellular localization of SNX1 and SNX2 from the Golgi area to an endosomal localization. A similar phenotype was previously shown for SNX1 after EHD3 knockdown [74], and also in these cells StxB accumulated in endosomes.
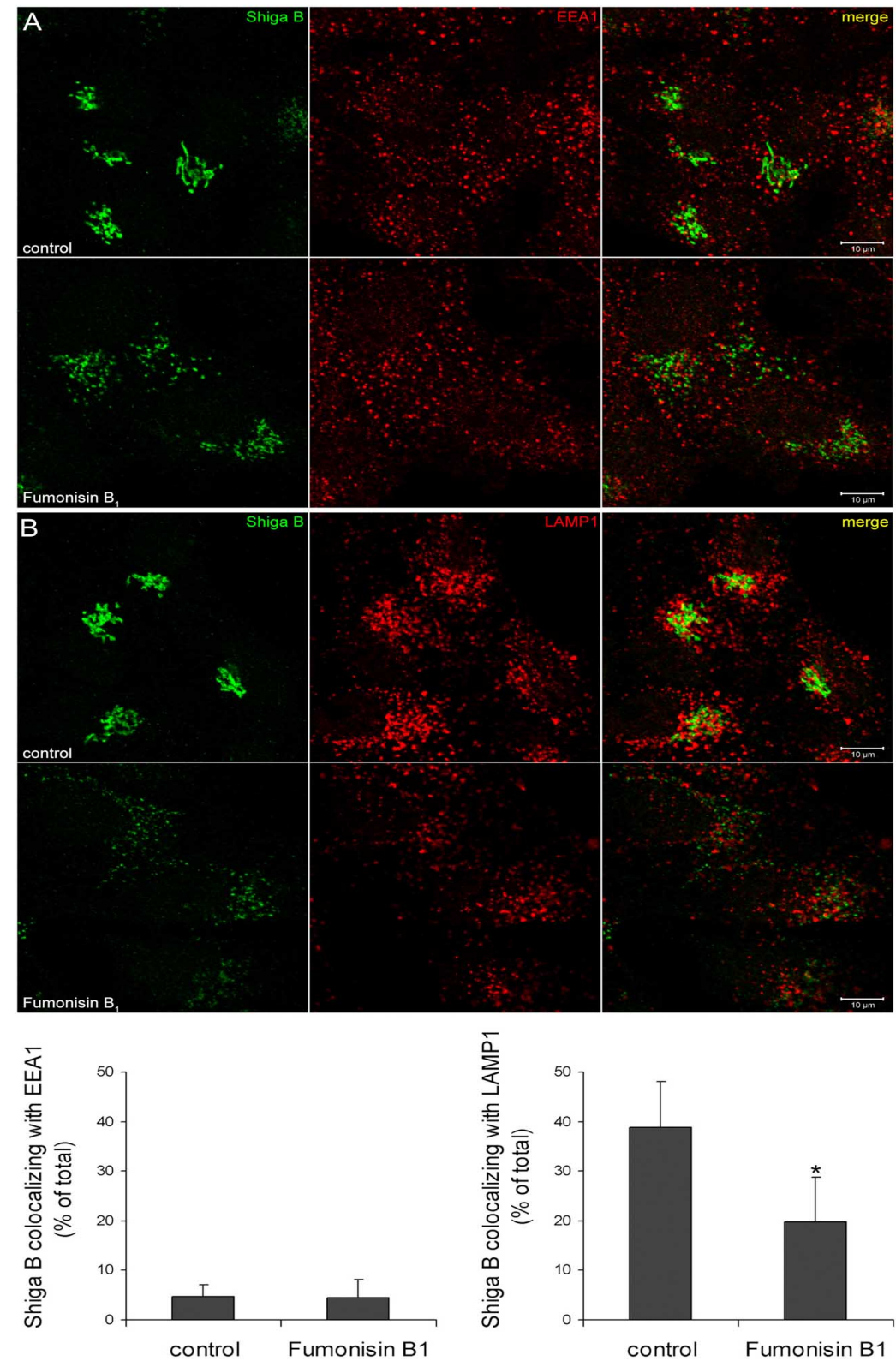

Fig. (3). StxB colocalization with lysosomes is not increased upon inhibition of Golgi transport with fumonisin $B_{1}$. The amount of receptor-bound Stx transported to the Golgi apparatus is reduced in HEp-2 cells pre-treated with $10 \mu \mathrm{M}$ fumonisin $\mathrm{B}_{1}$ for 48 hours. After 45 minutes of StxB incubation, the colocalization of StxB with early endosomal marker EEA1 (A) and lysosomal marker LAMP1 (B) does not increase in fumonisin $B_{1}$-treated cells compared to control cells. In the case of LAMP1 the colocalization rather decreases. $((A) n=27$ for both control and fumonisin $\mathrm{B}_{1},(\mathrm{~B}) \mathrm{n}=21$ for control and $\mathrm{n}=14$ for fumonisin $\mathrm{B}_{1},{ }^{*} \mathrm{P}<0.05$, unpaired Student's t-test). This suggests that in cells treated with fumonisin $\mathrm{B}_{1}$, StxB is retained in endosomes or transport vesicles unable to fuse with the Golgi apparatus rather than in EEA1- or LAMP1-positive endosomes or lysosomes. 
Inhibition of Stx trafficking and accumulation of the toxin in endosomes has also been shown when PKC $\delta$ [32] or p38 $\alpha$ [33] are inhibited or depleted from cells. Interestingly, both kinases are rapidly activated by Stx binding, further strengthening the hypothesis that Stx is able to stimulate its own trafficking by inducing signaling cascades. Activated p38 $\alpha$ was shown to translocate to endosomes upon Stx stimulation. Recently, potential modulators of the p38 $\alpha$ dependent pathway were identified, the $\beta$-arrestins 1 and 2 [95]. Like p38 $\alpha$, the $\beta$-arrestins were shown to translocate to endosomes upon Stx stimulation. However, the $\beta$-arrestins seem to be negative regulators of retrograde transport, as knockdown of these proteins increased the retrograde transport of both Stx and the MPRs [95]. It was proposed that since $\beta$-arrestins can form a complex with p38 they may sense the activated p38 and attenuate its signaling.

\section{GOLGI-TO-ER TRANSPORT}

ER-resident proteins that are missorted to the Golgi complex by anterograde transport, are normally retrieved by a retrograde transport system through the Golgi cisternae and back to the ER. The classical eukaryotic signal for retension in the ER lumen is the KDEL-motif, and proteins containing this motif are retrieved in COPI-coated vesicles by KDEL receptors [96, 97]. The Pseudomonas exotoxin A contains a KDEL-like sequence and exploits this pathway [98]. Also the cholera toxin A-chain contains a KDEL-motif, however, this motif is not strictly required for cholera toxin function, but rather improves the efficiency of intoxication [99]. Importantly, even the cholera toxin B-moiety can move retrogradely to the ER, demonstrating that the GM1-bound toxin can be transported independently of the A-chain containing the KDEL motif. Studies on Stx transport provided the first evidence that a bacterial protein was able to move retrogradely all the way from the plasma membrane to the ER [91], and a few years later this was also demonstrated to be the case for cholera toxin [100]. Interestingly, Stx does not have a KDEL motif and reaches the ER in a COPI-independent pathway [101]. Golgi-to-ER transport of Stx is dependent on Rab6a', actin, Cdc42, microtubuli and calcium [63, 102-105], and the vesicle trafficking seems to be mediated by myosin motors [106].

\section{TRANSLOCATION TO THE CYTOSOL}

In order to inhibit protein synthesis, the catalytically active $A_{1}$-chain of Stx must enter the cytosol, where the $28 \mathrm{~S}$ rRNA substrate is located. Several lines of evidence indicate that $\mathrm{Stx}_{1}$, like many other ER-targeted $\mathrm{AB}$ or $\mathrm{AB}_{5}$ toxins (e.g. ricin, cholera toxin, and Pseudomonas exotoxin A) translocates to the cytosol from the ER. ER-to-cytosol translocation is presumably achieved by taking advantage of a process known as ER-associated degradation (ERAD), which normally functions to dislocate misfolded proteins from the ER to the cytosol for immediate ubiquitylation and proteasomal degradation [107]. Stx, and other ER-directed toxins, avoid massive ubiquitylation and degradation at least in part due to the extremely low content of lysine residues in their A-moieties [108].

In cells containing furin, the Stx A-chain is already proteolytically cleaved before ER arrival [3], so that the $A_{1}$ fragment is connected to the $A_{2}$ fragment (which again is bound to the pentameric StxB moiety) only by a disulfide bridge between cysteines 242 and 261. For full catalytic activity, the $A_{1}$ fragment must presumably dissociate from $\mathrm{A}_{2}$, since residues 258-262 of $\mathrm{A}_{2}$ lie adjacent to the active site cleft, and the side chain of Met $_{260}$ protrudes into the active site itself [109]. Removal of the disulfide bridge is sufficient for the $A_{1}$ fragment to dissociate from the $A_{2}$ StxB-Gb3 complex [5]. How the disulfide bridge is reduced is not known, but in analogy with cholera toxin, whose active $A_{1}$ fragment also needs to be cleaved from its disulfide-linked $\mathrm{A}_{2}$ fragment, it is possible that the reduction also of StxA is carried out by the ER-resident enzyme protein disulfide isomerase [110,111]. In Vero cells (which contain furin) it was demonstrated that the vast majority of StxA in the cells was not reduced and only the $A_{1}$ chain could be detected in the cytosol [112]. However, it can not be excluded that also the whole A-chain can be translocated from the ER to the cytosol. Indeed, it has been reported that in vitro translated Stx A-chain is able to translocate from the lumen of isolated microsomal membranes [113]. Moreover, in Vero cells a Stx-mutant that lacks the furin cleavage site was shown to be processed in a manner that was sensitive to inhibitors of the cytosolic enzyme calpain [114], indicating that also in vivo, the whole A-chain may translocate to the cytosol. Given that the A-chain stays attached to the pentameric B-moiety (which again is most likely still bound to Gb3) all the way to the ER [112], it remains to be demonstrated how this intoxication process occurs.

The molecular mechanism responsible for $\mathrm{StxA}_{1^{-}}$ translocation from the ER to the cytosol is not well defined. In yeast, it was shown that the stretch consisting of the 12 very last C-terminal amino acid residues in $\mathrm{A}_{1}(240-251)$ is required for its ER-to-cytosol translocation [115]. Moreover, $\mathrm{A}_{1}$ contains a hydrophobic region (residues 224-242) close to its C-terminus, which may be recognized by ER-resident chaperones mediating ER quality control [108], but experimental data to support this idea are lacking. It seems evident, however, that both the A- and B-chains of Stx can interact with ER-resident chaperones. In vitro translated StxA was shown to interact with the ER-resident Hsp40 chaperone HEDJ/ERdj3 inside the lumen of microsomes isolated from Vero cells [113], and interestingly the ERresident Hsp70 chaperone BiP, which is likely to play a major role in ERAD substrate selection [107], was found to coimmunoprecipitate with StxA and HEDJ/ERdj3. More recently, a functional interplay between HEDJ/ERdj3 and $\mathrm{BiP}$ has been uncovered [116, 117]. Intriguingly, it appears that not only StxA, but also StxB is able to interact with BiP, since StxB has been reported to both colocalize [87] and coimmunoprecipitate with $\mathrm{BiP}$ [118]. Interestingly, $\mathrm{BiP}$ seems to play a central role not only in ERAD substrate selection, but possibly also in the targeting of ERAD substrates to an ER retrotranslocon channel [107]. It remains to be determined whether BiP actually both recognizes and drives StxA/StxA $A_{1}$ to an ER retrotranslocon channel. Furthermore, the nature of the retrotranslocon channel(s) used by StxA/StxA $A_{1}$ remains to be defined. One possibility is that Stx uses the same retrotranslocon (Sec61) as is apparently being exploited by cholera toxin and ricin to gain access to the cytosol $[119,120]$. However, it is unclear whether StxA can interact with Sec61 or not $[112,113]$. Alternatively, Stx might use other putative ER 
retrotranslocon channels, such as those constituted by the derlin family.

\section{EXPLOITATION OF STX IN MEDICINE}

The use of Shiga toxin, or the binding part of the toxin, has great potential in cancer diagnostics and treatment (for reviews see [7, 121-123]). The Stx receptor Gb3 has a limited expression in normal human tissues, being mostly restricted to endothelial cells, kidney epithelium and some antigen presenting cells, such as subsets of dendritic cells and B cells [11]. This facilitates the use of StxB as a vector for peptide delivery to the MHC class I pathway for the development of vaccines against specific cancer epitopes (for reviews see [121, 123]). It has been shown that exogenous antigens coupled to StxB is targeted to the MHC class I pathway and then presented on the surface of dendritic cells, facilitating activation of cytotoxic T-cells and antitumor immunity [124-126]. In this way StxB might function as a non-live, non-toxic vaccine delivery system in cancer therapy.

Interestingly, $\mathrm{Gb} 3$ is overexpressed in several cancer types, such as B-cell lymphomas, and cancers of the ovary, breast, testis, and colon (reviewed in [123]). For diagnostic purposes and selective imaging of these Gb3-expressing cells, visualizing agents, such as radioactive isotopes, contrast agents or fluorescent dyes, might be coupled to StxB. It has been demonstrated that labeled StxB administered systemically in mouse models accumulates in tumor regions overexpressing $\mathrm{Gb} 3$, thus confirming that cancer cells can be targeted in vivo by $\operatorname{StxB}[124,127]$. Although not yet tested in vivo, the coupling of chemotherapeutic drugs to StxB may increase the selectivity of chemotherapy, and thereby reduce side-effects. When a topoisomerase I inhibitor prodrug was coupled to StxB, the complex was targeted to the biosynthetic/secretory pathway (most likely the ER) and exhibited cytotoxicity only in Gb3positive cells [128].

There is also a potential use of the holotoxin in cancer treatment. It has been shown that ovarian-derived tumor cells were effectively killed by Stx1, and interestingly, multidrug resistant variants of these tumor cells expressed higher levels of Gb3 and were more sensitive to Stx1 [129]. Gb3 was expressed also in tumor vasculature, suggesting a potential role for Stx as an antiangiogenic agent [130]. Also some brain tumor cells, such as astrocytoma cells, are sensitive to Stx 1 and are killed by apoptosis [131]. Moreover, Stx 1 has proven useful as an ex-vivo purging agent to eradicate malignant cells from autologous stem cell grafts [132]. The Stx B-moiety has been demonstrated to reduce tumor growth of colon carcinoma cells in a mouse model [133].

\section{CONCLUDING REMARKS}

The fact that many protein toxins follow long intracellular transport routes in order to reach the cytosol, makes them invaluable tools in the study of cellular pathways. The investigations of toxin uptake and intracellular transport have given important insight in basic cell biology processes and led to the discovery of several transport pathways. Clearly, detailed knowledge of the regulation and the specific intracellular trafficking mechanisms used by the different toxins is crucial both to improve the prevention and treatment of toxin-induced diseases, and also to exploit the toxins as tools in medicine.

\section{ACKNOWLEDGEMENTS}

This work was supported by the Norwegian Cancer Society, the Norwegian Research Council for Science and the Humanities, and the Helse Sør-Øst, Norway.

\section{REFERENCES}

[1] Soltyk AM, MacKenzie CR, Wolski VM, et al. A mutational analysis of the globotriaosylceramide-binding sites of verotoxin VT1. J Biol Chem 2002; 277: 5351-9.

[2] Sandvig K. Shiga toxins. Toxicon 2001; 39: 1629-35.

[3] Garred $\varnothing$, van Deurs B, Sandvig K. Furin-induced cleavage and activation of Shiga toxin. J Biol Chem 1995; 270: 10817-21.

[4] Chiron MF, Fryling CM, FitzGerald DJ. Cleavage of pseudomonas exotoxin and diphtheria toxin by a furin-like enzyme prepared from beef liver. J Biol Chem 1994; 269: 18167-76.

[5] Garred $\varnothing$, Dubinina E, Polesskaya A, et al. Role of the disulfide bond in Shiga toxin A-chain for toxin entry into cells. J Biol Chem 1997; 272: 11414-9.

[6] O'Brien AD, Tesh VL, Donohue-Rolfe A, et al. Shiga toxin: biochemistry, genetics, mode of action, and role in pathogenesis. Curr Top Microbiol Immunol 1992; 180: 65-94.

[7] Sandvig K, van Deurs B. Delivery into cells: lessons learned from plant and bacterial toxins. Gene Ther 2005; 12: 865-72.

[8] Sandvig K. In: Alouf J, Popoff M, Eds. The Comprehensive Sourcebook of Bacterial Protein Toxins Academic Press: Paris 2006; 310-22.

[9] Tam P, Mahfoud R, Nutikka A, et al. Differential intracellular transport and binding of verotoxin 1 and verotoxin 2 to globotriaosylceramide-containing lipid assemblies. J Cell Physiol 2008; 216: 750-63.

[10] Karmali MA. Infection by Shiga toxin-producing Escherichia coli: an overview. Mol Biotechnol 2004; 26: 117-22.

[11] Smith DC, Lord JM, Roberts LM, Johannes L. Glycosphingolipids as toxin receptors. Semin Cell Dev Biol 2004; 15: 397-408.

[12] Tetaud C, Falguières T, Carlier K, et al. Two distinct Gb3/CD77 signaling pathways leading to apoptosis are triggered by antiGb3/CD77 mAb and verotoxin-1. J Biol Chem 2003; 278: 45200-8.

[13] van de Kar NC, Monnens LA, van Hinsbergh VW. Tumor necrosis factor and interleukin 1 induce expression of the glycolipid verotoxin receptor in human endothelial cells. Implications for the pathogenesis of the haemolytic uraemic syndrome. Behring Inst Mitt 1993; (92): 202-9.

[14] Meyers KE, Kaplan BS. Many cell types are Shiga toxin targets. Kidney Int 2000; 57: 2650-1.

[15] Cherla RP, Lee SY, Tesh VL. Shiga toxins and apoptosis. FEMS Microbiol Lett 2003; 228: 159-66.

[16] Gordon J, Challa A, Levens JM, et al. CD40 ligand, Bcl-2, and Bcl-xL spare group I Burkitt lymphoma cells from CD77-directed killing via Verotoxin-1 B chain but fail to protect against the holotoxin. Cell Death Differ 2000; 7: 785-94.

[17] Head SC, Karmali MA, Lingwood CA. Preparation of VT1 and VT2 hybrid toxins from their purified dissociated subunits. Evidence for B subunit modulation of a subunit function. J Biol Chem 1991; 266: 3617-21.

[18] Nakajima H, Kiyokawa N, Katagiri YU, et al. Kinetic analysis of binding between Shiga toxin and receptor glycolipid Gb3Cer by surface plasmon resonance. J Biol Chem 2001; 276: 42915-22.

[19] Binnington B, Lingwood D, Nutikka A, Lingwood CA. Effect of globotriaosyl ceramide fatty acid alpha-hydroxylation on the binding by verotoxin 1 and verotoxin 2 . Neurochem Res 2002; 27: 807-13.

[20] Chark D, Nutikka A, Trusevych N, Kuzmina J, Lingwood C. Differential carbohydrate epitope recognition of globotriaosyl ceramide by verotoxins and a monoclonal antibody. Eur J Biochem 2004; 271: 405-17.

[21] Fraser ME, Fujinaga M, Cherney MM, et al. Structure of shiga toxin type 2 (Stx2) from Escherichia coli O157:H7. J Biol Chem 2004; 279: 27511-7.

[22] Kiarash A, Boyd B, Lingwood CA. Glycosphingolipid receptor function is modified by fatty acid content. Verotoxin 1 and verotoxin 2c preferentially recognize different globotriaosyl 
ceramide fatty acid homologues. J Biol Chem 1994; 269: 1113846.

[23] Arab S, Lingwood CA. Influence of phospholipid chain length on verotoxin/globotriaosyl ceramide binding in model membranes: comparison of a supported bilayer film and liposomes. Glycoconj J 1996; 13: 159-66.

[24] Mahfoud R, Manis A, Lingwood CA. Fatty acid-dependent globotriaosyl ceramide receptor function in detergent resistant model membranes. J Lipid Res 2009; 50(9): 1744-55.

[25] Khan F, Proulx F, Lingwood CA. Detergent-resistant globotriaosyl ceramide may define verotoxin/glomeruli-restricted hemolytic uremic syndrome pathology. Kidney Int 2009; 75(11): 1135-7.

[26] Hanashima T, Miyake M, Yahiro K, et al. Effect of Gb3 in lipid rafts in resistance to Shiga-like toxin of mutant Vero cells. Microb Pathog 2008; 45: 124-33.

[27] Falguières T, Romer W, Amessou M, et al. Functionally different pools of Shiga toxin receptor, globotriaosyl ceramide, in $\mathrm{HeLa}$ cells. FEBS J 2006; 273(22): 5205-18.

[28] Katagiri YU, Mori T, Nakajima H, et al. Activation of Src family kinase yes induced by Shiga toxin binding to globotriaosyl ceramide (Gb3/CD77) in low density, detergent-insoluble microdomains. J Biol Chem 1999; 274: 35278-82.

[29] Murata K, Higuchi T, Takada K, et al. Verotoxin-1 stimulation of macrophage-like THP-1 cells up-regulates tissue factor expression through activation of c-Yes tyrosine kinase: Possible signal transduction in tissue factor up-regulation. Biochim Biophys Acta 2006; 1762: 835-43.

[30] Mori T, Kiyokawa N, Katagiri YU, et al. Globotriaosyl ceramide $(\mathrm{CD} 77 / \mathrm{Gb} 3)$ in the glycolipid-enriched membrane domain participates in B-cell receptor-mediated apoptosis by regulating lyn kinase activity in human B cells. Exp Hematol 2000; 28: 1260-8.

[31] Lauvrak SU, Wälchli S, Iversen T-G, et al. Shiga toxin regulates its entry in a Syk-dependent manner. Mol Biol Cell 2006; 17: 1096109.

[32] Torgersen ML, Wälchli S, Grimmer S, Skånland SS, Sandvig K. Protein kinase Cdelta is activated by Shiga toxin and regulates its transport. J Biol Chem 2007; 282: 16317-28.

[33] Wälchli S, Skånland SS, Gregers TF, et al. The Mitogen-activated protein kinase p38 links Shiga Toxin-dependent signaling and trafficking. Mol Biol Cell 2008; 19: 95-104.

[34] Sandvig K, Olsnes S, Brown JE, Petersen OW, van Deurs B. Endocytosis from coated pits of Shiga toxin: a glycolipid-binding protein from Shigella dysenteriae 1. J Cell Biol 1989; 108: 133143.

[35] Torgersen ML, Lauvrak SU, Sandvig K. The A-subunit of surfacebound Shiga toxin stimulates clathrin-dependent uptake of the toxin. FEBS J 2005; 272: 4103-13.

[36] Shimizu T, Hamabata T, Yoshiki A, et al. An association of 27and $40-\mathrm{kDa}$ molecules with glycolipids that bind A-B bacterial enterotoxins to cultured cells. Biochim Biophys Acta 2003; 1612: 186-94.

[37] Wälchli S, Aasheim HC, Skånland SS, et al. Characterization of clathrin and Syk interaction upon Shiga toxin binding. Cell Signal 2009; 21(7): 1161-8.

[38] Takenouchi H, Kiyokawa N, Taguchi T, et al. Shiga toxin binding to globotriaosyl ceramide induces intracellular signals that mediate cytoskeleton remodeling in human renal carcinoma-derived cells. J Cell Sci 2004; 117: 3911-22.

[39] Hehnly H, Sheff D, Stamnes M. Shiga toxin facilitates its retrograde transport by modifying microtubule dynamics. Mol Biol Cell 2006; 17: 4379-89.

[40] Lauvrak SU, Torgersen ML, Sandvig K. Efficient endosome-toGolgi transport of Shiga toxin is dependent on dynamin and clathrin. J Cell Sci 2004; 117: 2321-31.

[41] Schapiro FB, Lingwood C, Furuya W, Grinstein S. pH-independent retrograde targeting of glycolipids to the Golgi complex. Am J Physiol 1998; 274: C319-C332.

[42] Khine AA, Lingwood CA. Capping and receptor-mediated endocytosis of cell-bound verotoxin (Shiga-like toxin). 1: Chemical identification of an amino acid in the B subunit necessary for efficient receptor glycolipid binding and cellular internalization. J Cell Physiol 1994; 161: 319-32.

[43] Nichols BJ, Kenworthy AK, Polishchuk RS, et al. Rapid cycling of lipid raft markers between the cell surface and golgi complex. J Cell Biol 2001; 153: 529-42.
[44] Saint-Pol A, Yelamos B, Amessou M, et al. Clathrin adaptor epsinR is required for retrograde sorting on Early Endosomal Membranes. Dev Cell 2004; 6: 525-38.

[45] Römer W, Berland L, Chambon V, et al. Shiga toxin induces tubular membrane invaginations for its uptake into cells. Nature 2007; 450: 670-5.

[46] Damke H, Baba T, van der Bliek AM, Schmid SL. Clathrinindependent pinocytosis is induced in cells overexpressing a temperature-sensitive mutant of dynamin. J Cell Biol 1995; 131: 69-80.

[47] Johannes L, Popoff V. Tracing the retrograde route in protein trafficking. Cell 2008; 135: 1175-87.

[48] Mallard F, Antony C, Tenza D, et al. Direct pathway from early/recycling endosomes to the Golgi apparatus revealed through the study of shiga toxin B-fragment transport. J Cell Biol 1998; 143: 973-90.

[49] Ghosh RN, Mallet WG, Soe TT, McGraw TE, Maxfield FR. An endocytosed TGN38 chimeric protein is delivered to the TGN after trafficking through the endocytic recycling compartment in $\mathrm{CHO}$ cells. J Cell Biol 1998; 142: 923-36.

[50] Mallet WG, Maxfield FR. Chimeric forms of furin and TGN38 are transported with the plasma membrane in the trans-Golgi network via distinct endosomal pathways. J Cell Biol 1999; 146: 345-59.

[51] Seaman MN. Cargo-selective endosomal sorting for retrieval to the Golgi requires retromer. J Cell Biol 2004; 165: 111-22.

[52] Natarajan R, Linstedt AD. A cycling cis-Golgi protein mediates endosome-to-Golgi traffic. Mol Biol Cell 2004; 15: 4798-806.

[53] Spooner RA, Smith DC, Easton AJ, Roberts LM, Lord JM. Retrograde transport pathways utilised by viruses and protein toxins. Virol J 2006; 3: 26.

[54] Lombardi D, Soldati T, Riederer MA, et al. Rab9 functions in transport between late endosomes and the trans Golgi network. EMBO J 1993; 12: 677-82.

[55] Sandvig K, Grimmer S, Lauvrak U, et al. Pathways followed by ricin and Shiga toxin into cells. Histochem Cell Biol 2002; 117: $131-41$.

[56] Iversen T-G, Skretting G, Llorente A, et al. Endosome to Golgi transport of ricin is independent of clathrin and of the Rab9- and Rab11-GTPases. Mol Biol Cell 2001; 12: 2099-107.

[57] Utskarpen A, Slagsvold HH, Iversen T-G, Wälchli S, Sandvig K. Transport of ricin from endosomes to the Golgi apparatus is regulated by Rab6A and Rab6A'. Traffic 2006; 7: 663-72.

[58] Lauvrak SU, Llorente A, Iversen T-G, Sandvig K. Selective regulation of the Rab9-independent transport of ricin to the Golgi apparatus by calcium. J Cell Sci 2002; 115: 3449-56.

[59] Mallard F, Tang BL, Galli T, et al. Early/recycling endosomes-toTGN transport involves two SNARE complexes and a Rab6 isoform. J Cell Biol 2002; 156: 653-64.

[60] Wilcke M, Johannes L, Galli $\mathrm{T}$, et al. Rab11 regulates the compartmentalization of early endosomes required for efficient transport from early endosomes to the trans-golgi network. J Cell Biol 2000; 151: 1207-20.

[61] Lieu ZZ, Derby MC, Teasdale RD, et al. The golgin GCC88 is required for efficient retrograde transport of cargo from the early endosomes to the trans-Golgi network. Mol Biol Cell 2007; 18: 4979-91.

[62] Raa H, Grimmer S, Schwudke D, et al. Glycosphingolipid requirements for endosome-to-Golgi transport of Shiga toxin. Traffic 2009; 10(7): 868-82.

[63] Del Nery E, Miserey-Lenkei S, Falguières T, et al. Rab6A and Rab6A' GTPases play non-overlapping roles in membrane trafficking. Traffic 2006; 7: 394-407.

[64] Monier S, Jollivet F, Janoueix-Lerosey I, Johannes L, Goud B. Characterization of novel Rab6-interacting proteins involved in endosome-to-TGN transport. Traffic 2002; 3: 289-97.

[65] Fuchs E, Haas AK, Spooner RA, et al. Specific Rab GTPaseactivating proteins define the Shiga toxin and epidermal growth factor uptake pathways. J Cell Biol 2007; 177: 1133-43.

[66] Llorente A, Rapak A, Schmid SL, van Deurs B, Sandvig K. Expression of mutant dynamin inhibits toxicity and transport of endocytosed ricin to the Golgi apparatus. J Cell Biol 1998; 140: 553-63.

[67] Bujny MV, Popoff V, Johannes L, Cullen PJ. The retromer component sorting nexin-1 is required for efficient retrograde transport of Shiga toxin from early endosome to the trans Golgi network. J Cell Sci 2007; 120: 2010-21. 
[68] Utskarpen A, Slagsvold HH, Dyve AB, Skånland SS, Sandvig K. SNX1 and SNX2 mediate retrograde transport of Shiga toxin. Biochem Biophys Res Commun 2007; 358: 566-70.

[69] Popoff V, Mardones GA, Tenza D, et al. The retromer complex and clathrin define an early endosomal retrograde exit site. J Cell Sci 2007; 120: 2022-31.

[70] Bonifacino JS, Hurley JH. Retromer. Curr Opin Cell Biol 2008; 20: 427-36.

[71] Collins BM. The structure and function of the retromer protein complex. Traffic 2008; 9: 1811-22.

[72] Seaman MN. Recycle your receptors with retromer. Trends Cell Biol 2005; 15: 68-75.

[73] Skånland SS, Wälchli S, Utskarpen A, Wandinger-Ness A, Sandvig K. Phosphoinositide-regulated retrograde transport of ricin: crosstalk between hVps34 and sorting nexins. Traffic 2007; 8: 297 309.

[74] Naslavsky N, McKenzie J, tan-Bonnet N, Sheff D, Caplan S. EHD3 regulates early-endosome-to-Golgi transport and preserves Golgi morphology. J Cell Sci 2009; 122: 389-400.

[75] Yoshino A, Setty SR, Poynton C, et al. tGolgin-1 (p230, golgin245) modulates Shiga-toxin transport to the Golgi and Golgi motility towards the microtubule-organizing centre. J Cell Sci 2005; 118: 2279-93.

[76] Lu L, Tai G, Hong W. Autoantigen Golgin-97, an effector of Arl1 GTPase, participates in traffic from the endosome to the trans-golgi network. Mol Biol Cell 2004; 15: 4426-43.

[77] Nishimoto-Morita K, Shin HW, Mitsuhashi H, et al. Differential effects of depletion of ARL1 and ARFRP1 on membrane trafficking between the trans-Golgi network and endosomes. J Biol Chem 2009; 284(16): 10583-92.

[78] Zolov SN, Lupashin VV. Cog3p depletion blocks vesicle-mediated Golgi retrograde trafficking in HeLa cells. J Cell Biol 2005; 168 : 747-59.

[79] Derby MC, Lieu ZZ, Brown D, et al. The trans-Golgi network golgin, GCC185, is required for endosome-to-Golgi transport and maintenance of Golgi structure. Traffic 2007; 8: 758-73.

[80] Perez-Victoria FJ, Mardones GA, Bonifacino JS. Requirement of the human GARP complex for mannose 6-phosphate-receptordependent sorting of cathepsin D to lysosomes. Mol Biol Cell 2008; 19: 2350-62.

[81] Yamane J, Kubo A, Nakayama K, et al. Functional involvement of TMF/ARA160 in Rab6-dependent retrograde membrane traffic. Exp Cell Res 2007; 313: 3472-85.

[82] Tai G, Lu L, Wang TL, et al. Participation of the syntaxin 5/Ykt6/GS28/GS15 SNARE complex in transport from the early/recycling endosome to the trans-Golgi network. Mol Biol Cell 2004; 15: 4011-22.

[83] Le PU, Nabi IR. Distinct caveolae-mediated endocytic pathways target the Golgi apparatus and the endoplasmic reticulum. J Cell Sci 2003; 116: 1059-71.

[84] Orlandi PA, Fishman PH. Filipin-dependent inhibition of cholera toxin: evidence for toxin internalization and activation through caveolae-like domains. J Cell Biol 1998; 141: 905-15.

[85] Puri V, Watanabe R, Singh RD, et al. Clathrin-dependent and independent internalization of plasma membrane sphingolipids initiates two Golgi targeting pathways. J Cell Biol 2001; 154: 53547.

[86] Wolf AA, Fujinaga Y, Lencer WI. Uncoupling of the cholera toxinG(M1) ganglioside receptor complex from endocytosis, retrograde Golgi trafficking, and downstream signal transduction by depletion of membrane cholesterol. J Biol Chem 2002; 277: 16249-56.

[87] Falguières T, Mallard F, Baron C, et al. Targeting of Shiga toxin Bsubunit to retrograde transport route in association with detergentresistant membranes. Mol Biol Cell 2001; 12: 2453-68.

[88] Kovbasnjuk O, Edidin M, Donowitz M. Role of lipid rafts in Shiga toxin 1 interaction with the apical surface of Caco-2 cells. J Cell Sci 2001; 114: 4025-31.

[89] Fujinaga Y, Wolf AA, Rodighiero C, et al. Gangliosides that associate with lipid rafts mediate transport of cholera and related toxins from the plasma membrane to endoplasmic reticulm. Mol Biol Cell 2003; 14: 4783-93.

[90] Hoey DE, Sharp L, Currie C, et al. Verotoxin 1 binding to intestinal crypt epithelial cells results in localization to lysosomes and abrogation of toxicity. Cell Microbiol 2003; 5: 85-97.

[91] Sandvig K, Garred $\varnothing$, Prydz K, et al. Retrograde transport of endocytosed Shiga toxin to the endoplasmic reticulum. Nature 1992; 358: 510-2.
[92] Sandvig K, Garred Ø, van Helvoort A, van Meer G, van Deurs B. Importance of glycolipid synthesis for butyric acid-induced sensitization to Shiga toxin and intracellular sorting of toxin in A431 cells. Mol Biol Cell 1996; 7: 1391-404.

[93] Arab S, Lingwood CA. Intracellular targeting of the endoplasmic reticulum/nuclear envelope by retrograde transport may determine cell hypersensitivity to verotoxin via globotriaosyl ceramide fatty acid isoform traffic. J Cell Physiol 1998; 177: 646-60.

[94] Sandvig K, Ryd M, Garred $\varnothing$, et al. Retrograde transport from the Golgi complex to the ER of both shiga toxin and the nontoxic shiga B-fragment is regulated by butyric acid and cAMP. J Cell Biol 1994; 126: 53-64.

[95] Skånland SS, Wälchli S, Sandvig K. beta-arrestins attenuate p38 mediated endosome to Golgi transport. Cell Microbiol 2009; [Epub ahead of print]

[96] Warren G, Malhotra V. The organisation of the Golgi apparatus. Curr Opin Cell Biol 1998; 10: 493-8.

[97] Munro S, Pelham HR. A C-terminal signal prevents secretion of luminal ER proteins. Cell 1987; 48: 899-907.

[98] Jackson ME, Simpson JC, Girod A, et al. The KDEL retrieval system is exploited by Pseudomonas exotoxin A, but not by Shigalike toxin-1, during retrograde transport from the Golgi complex to the endoplasmic reticulum. J Cell Sci 1999; 112 ( Pt 4): 467-75.

[99] Lencer WI, Constable C, Moe S, et al. Targeting of cholera toxin and Escherichia coli heat labile toxin in polarized epithelia: role of COOH-terminal KDEL. J Cell Biol 1995; 131: 951-62.

[100] Sandvig K, Garred $\varnothing$, van Deurs B. Thapsigargin-induced transport of cholera toxin to the endoplasmic reticulum. Proc Natl Acad Sci USA 1996; 93: 12339-43.

[101] Girod A, Storrie B, Simpson JC, et al. Evidence for a COP-Iindependent transport route from the Golgi complex to the endoplasmic reticulum. Nat Cell Biol 1999; 1: 423-30.

[102] Valderrama F, Duran JM, Babia T, et al. Actin microfilaments facilitate the retrograde transport from the Golgi complex to the endoplasmic reticulum in mammalian cells. Traffic 2001; 2: 717 26.

[103] Luna A, Matas OB, Martinez-Menarguez JA, et al. Regulation of protein transport from the Golgi complex to the endoplasmic reticulum by CDC42 and N-WASP. Mol Biol Cell 2002; 13: 86679.

[104] Chen JL, Ahluwalia JP, Stamnes M. Selective effects of calcium chelators on anterograde and retrograde protein transport in the cell. J Biol Chem 2002; 277: 35682-7.

[105] Pernet-Gallay K, Antony C, Johannes L, et al. The overexpression of GMAP-210 blocks anterograde and retrograde transport between the ER and the Golgi apparatus. Traffic 2002; 3: 822-32.

[106] Duran JM, Valderrama F, Castel S, et al. Myosin motors and not actin comets are mediators of the actin-based Golgi-to-endoplasmic reticulum protein transport. Mol Biol Cell 2003; 14: 445-59.

[107] Vembar SS, Brodsky JL. One step at a time: endoplasmic reticulum-associated degradation. Nat Rev Mol Cell Biol 2008; 9: 944-57.

[108] Hazes B, Read RJ. Accumulating evidence suggests that several AB-toxins subvert the endoplasmic reticulum-associated protein degradation pathway to enter target cells. Biochemistry 1997; 36: 11051-4.

[109] Fraser ME, Chernaia MM, Kozlov YV, James MN. Crystal structure of the holotoxin from Shigella dysenteriae at $2.5 \mathrm{~A}$ resolution. Nat Struct Biol 1994; 1: 59-64.

[110] Tsai B, Rodighiero C, Lencer WI, Rapoport TA. Protein disulfide isomerase acts as a redox-dependent chaperone to unfold cholera toxin. Cell 2001; 104: 937-48.

[111] Tsai B, Rapoport TA. Unfolded cholera toxin is transferred to the ER membrane and released from protein disulfide isomerase upon oxidation by Ero1. J Cell Biol 2002; 159: 207-16.

[112] Tam PJ, Lingwood CA. Membrane cytosolic translocation of verotoxin A1 subunit in target cells. Microbiology 2007; 153: 2700-10.

[113] Yu M, Haslam DB. Shiga toxin is transported from the endoplasmic reticulum following interaction with the luminal chaperone HEDJ/ERdj3. Infect Immun 2005; 73: 2524-32.

[114] Garred $\varnothing$, Dubinina E, Holm PK, et al. Role of processing and intracellular transport for optimal toxicity of Shiga toxin and toxin mutants. Exp Cell Res 1995; 218: 39-49.

[115] LaPointe P, Wei X, Gariépy J. A role for the protease-sensitive loop region of Shiga-like toxin 1 in the retrotranslocation of its A1 
domain from the endoplasmic reticulum lumen. J Biol Chem 2005; 280: $23310-8$.

[116] Shen Y, Hendershot LM. ERdj3, a stress-inducible endoplasmic reticulum DnaJ homologue, serves as a cofactor for BiP's interactions with unfolded substrates. Mol Biol Cell 2005; 16: 4050.

[117] Jin Y, Awad W, Petrova K, Hendershot LM. Regulated release of ERdj3 from unfolded proteins by BiP. EMBO J 2008; 27: 2873-82.

[118] Falguières T, Johannes L. Shiga toxin B-subunit binds to the chaperone BiP and the nucleolar protein B23. Biol Cell 2006; 98: 125-34.

[119] Schmitz A, Herrgen H, Winkeler A, Herzog V. Cholera toxin is exported from microsomes by the Sec61p complex. J Cell Biol 2000; 148: 1203-12.

[120] Simpson JC, Roberts LM, Romisch K, et al. Ricin A chain utilises the endoplasmic reticulum-associated protein degradation pathway to enter the cytosol of yeast. FEBS Lett 1999; 459: 80-4.

[121] Smith DC, Lord JM, Roberts LM, Tartour E, Johannes L. 1st class ticket to class I: protein toxins as pathfinders for antigen presentation. Traffic 2002; 3: 697-704.

[122] Johannes L, Decaudin D. Protein toxins: intracellular trafficking for targeted therapy. Gene Ther 2005; 12: 1360-8.

[123] Tarrago-Trani MT, Storrie B. Alternate routes for drug delivery to the cell interior: pathways to the Golgi apparatus and endoplasmic reticulum. Adv Drug Deliv Rev 2007; 59: 782-97.

[124] Janssen KP, Vignjevic D, Boisgard R, et al. In vivo tumor targeting using a novel intestinal pathogen-based delivery approach. Cancer Res 2006; 66: 7230-6.

[125] Vingert B, Adotevi O, Patin D, et al. The Shiga toxin B-subunit targets antigen in vivo to dendritic cells and elicits anti-tumor immunity. Eur J Immunol 2006; 36: 1124-35.
[126] Haicheur N, Benchetrit F, Amessou M, et al. The B subunit of Shiga toxin coupled to full-size antigenic protein elicits humoral and cell-mediated immune responses associated with a Th1dominant polarization. Int Immunol 2003; 15: 1161-71.

[127] Viel T, Dransart E, Nemati F, et al. In vivo tumor targeting by the B-subunit of shiga toxin. Mol Imaging 2008; 7: 239-47.

[128] El Alaoui A, Schmidt F, Amessou M, et al. Shiga toxin-mediated retrograde delivery of a topoisomerase I inhibitor prodrug. Angew Chem Int Ed Engl 2007; 46: 6469-72.

[129] Farkas-Himsley H, Hill R, Rosen B, Arab S, Lingwood CA. The bacterial colicin active against tumor cells in vitro and in vivo is verotoxin 1. Proc Natl Acad Sci USA 1995; 92: 6996-7000.

[130] Arab S, Russel E, Chapman WB, Rosen B, Lingwood CA. Expression of the verotoxin receptor glycolipid, globotriaosylceramide, in ovarian hyperplasias. Oncol Res 1997; 9 : 553-63.

[131] Arab S, Murakami M, Dirks P, et al. Verotoxins inhibit the growth of and induce apoptosis in human astrocytoma cells. J Neurooncol 1998; 40: 137-50.

[132] LaCasse EC, Bray MR, Patterson B, et al. Shiga-like toxin-1 receptor on human breast cancer, lymphoma, and myeloma and absence from $\mathrm{CD} 34(+)$ hematopoietic stem cells: implications for ex vivo tumor purging and autologous stem cell transplantation. Blood 1999; 94: 2901-10.

[133] Kovbasnjuk O, Mourtazina R, Baibakov B, et al. The glycosphingolipid globotriaosylceramide in the metastatic transformation of colon cancer. Proc Natl Acad Sci USA 2005; 102: $19087-92$

(C) Torgersen et al.; Licensee Bentham Open.

This is an open access article licensed under the terms of the Creative Commons Attribution Non-Commercial License (http: //creativecommons.org/licenses/by$\mathrm{nc} / 3.0 /$ ) which permits unrestricted, non-commercial use, distribution and reproduction in any medium, provided the work is properly cited. 